\title{
Introduction to the Minitrack on Emerging Issues in Distributed Group Decision-Making: Opportunities and Challenges
}

\author{
Anil K. Aggarwal \\ University of Baltimore \\ USA \\ aaggarwal@ubalt.edu
}

\author{
Doug Vogel \\ Harbin Institute of Technology \\ PRC \\ isdoug@cityu.edu.hk
}

\author{
Yuko Murayama \\ Tsuda College \\ Japan \\ murayama@tsuda.ac.jp
}

This mini track addresses emerging issues, such as diversity, culture, adaptability, mobility and agility related to teams in distributed group decision-making, as well as the underlying theories of group dynamics, coordination, and communications in both swift and ad-hoc groups. The papers submitted specifically examined the emerging issues related to team configuration, diversity and performance in a distributed environment.

The mini track attracted several papers related to various aspects of distributed decision making. Accepted papers study decision making during disaster recovery and multi-lateral diplomacy in a distributed environment. In the First paper authors contend Crisis in and around Ukraine is becoming the first show-case in multilateral diplomacy, where Remotely Piloted Aircraft Systems (RPAS) and other technologies are deployed to produce information for the diplomatic processes of the Organization for Security and Cooperation in Europe (OSCE) and to public at the same time. This has taken place in a very rapid manner without time for descent planning and through learning by doing before and in the middle of the start and development of the crisis special monitoring mission based on the consensus of 57 member states in a distributed decision making environment. This paper approaches these multilateral diplomacy scenes by looking at the work done through two disciplines which "orchestrate"; Social Science and Information Systems. In addition, authors stress Abbot et al. orchestration theory application into OSCE and to its "Ukraine toolbox" needs more research, in which e.g. IS literature's socio-technical modeling methods will be helpful in order to bridge recognized gaps from practice and literature.
In the second paper authors discuss Disaster information processing in context of Great East Japan Earthquake and Tsunami on March 11th, 2011 and misinformation spread around during the disaster. Their work was motivated to see the misinformation by twitter at the Great East Japan Earthquake and Tsunami in Japan in 2011. Author(s) study why people retweet and conducted user survey and found several factors like willingness to provide relevant information, subjective feelings and interest, and information they perceived to be important.

Each of the above paper is timely, as they address emerging issues related to distributed group decisionmaking. The distributed group decision making area is still emerging and research is conflicting. As long as research produces mixed results, there will be continual need for validation and replication of experiments and development of new underlying theories. 